\title{
Does being born big confer advantages?
}

\author{
Katherine Gray-Donald PhD
}

See related research article by Wassimi and colleagues, page 322

$\mathrm{H}$ ealth disparities are evident in Canada when comparing Aboriginal communities to the majority population, as Wassimi and colleagues point out in their study of perinatal and postneonatal mortality by birthweight. ${ }^{1}$ In a step toward understanding the higher infant mortality among First Nations communities, the authors tackled the interesting question of whether there are survival advantages to large-for-gestational-age birthweight. In other words, are big babies better survivors? The study focused on First Nations people living in Quebec. Only those who reported speaking their First Nations language were included because they were identified in this manner. This population was compared with infants of Quebecers whose mother tongue is French.

The Cree of northern Quebec have very large infants owing to high pregravid body weights and high incidence of gestational diabetes. ${ }^{2}$ The birthweight curve is shifted to the right compared with that for non-First Nations women. Whether this is a recent phenomenon is not clear. More importantly, the consequences for infant health in the first year of life are not well documented. In their follow-up analysis linking data on births and deaths up to one year of age, Wassimi and colleagues report very large differences in birthweight distribution between groups. Small-for-gestational-age births made up $4 \%$ in the First Nations group and $11 \%$ in the French-language group, and large-for-gestational-age births made up 28\% in the First Nations group and $8 \%$ in the French-language group.

On close examination of the relative death rates in the two populations, one sees that, among appropriate-for-gestational-age infants, the relative risks of mortality are much higher in the First Nations group for both perinatal death (RR 1.77) and postneonatal death (RR 4.28). The First Nations group is clearly disadvantaged, with higher death rates in the small-for-gestational-age group as well.

Interestingly, however, there is no such disadvantage when perinatal mortality of large-forgestational-age infants is compared between the
First Nations group and French-language control group. With only seven deaths in 10 years in the First Nations group, the effect is nonsignificant, and there is certainly no excess mortality. In fact, the lowest perinatal mortality within the First Nations group appears to be in the heaviest group. In contrast, large-for-gestational-age birthweight is a clear risk factor for perinatal mortality among infants of mothers whose mother tongue is French.

The findings for postneonatal mortality are complex and particularly intriguing. In the French-language group, there is a clear protective effect of large-for-gestational-age birthweight against postneonatal mortality, and this effect is even more evident when analysis is restricted to deaths caused by sudden infant death syndrome (SIDS). This advantage of large-for-gestational-age birthweight in regard to SIDS has also been observed in the United States, as reported by a US study linking infant birth and death certificates.

Among the First Nations infants, no such risk reduction for postneonatal mortality was seen. When one compares the First Nations outcomes by birthweight (within their own group), there is a very modest increase in postneonatal mortality and SIDS in the large-for-gestational-age group.

These two trends going in opposite directions would lead us to believe that First Nations infants who are born large for gestational age have a serious risk of postneonatal death. Certainly, First Nations infants are at higher risk of postneonatal mortality, but being heavy does not greatly exacerbate their high rates as one might conclude when comparing them to the large-for-

\section{KEY POINTS}

- Infants of First Nations mothers in Quebec have higher perinatal and postneonatal death rates compared to those of women whose mother tongue is French.

- Postneonatal death rates are particularly high among First Nations infants.

- Different patterns of risk for perinatal and postneonatal mortality are evident among large-for-gestational-age infants when comparing births to First Nations women versus women whose mother tongue is French. 
${ }^{\text {PrPRISTIQ }}{ }^{\circledast}$ is indicated for the symptomatic relief of major depressive disorder. The short-term efficacy of PRISTIQ (desvenlafaxine succinate extended-release tablets) has been demonstrated in placebo-controlled trials of up to 8 weeks.

The most commonly observed adverse events associated with the use of PRISTIQ (at an incidence $\geq 5 \%$ and at least twice the rate of placebo) were nousea $(22 \%)$, dizziness $(13 \%)$, hyperhidrosis $(10 \%)$, constipation $(9 \%)$, and decreased appetite $(5 \%)$.

PRISTIQ is not indicated for use in children under the age of 18 . PRISTIQ is contraindicated in patients taking monoamine oxidase inhibitors (MAOIs), including linezolid, an antibiotic, methylene blue, a dye used in certain surgeries, or in patients who have taken MAOIs within the preceding 14 days due to risk of serious, sometimes fatal, drug interactions with selective serotonin reuptake inhibitor (SSRI) or serotonin norepinephrine reuptake inhibitor (SNRI) treatment or with other serotonergic drugs. These interactions have been associated with symptoms that include tremor, myoclonus, diaphoresis, nousea, vomiting, flushing, dizziness, hyperthermia with features resembling neuroleptic malignant syndrome, seizures, rigidity, autonomic instability with possible rapid fluctuations of vital signs, and mental status changes that include extreme agitation progressing to delirium and coma. Based on the half-life of desvenlafaxine succinate, at least 7 days should be allowed after stopping desvenlafaxine succinate and before starting an MAOI.

PRISTIQ is contraindicated in patients demonstrating hypersensitivity to desvenlafaxine succinate extended-release, venlafaxine hydrochloride or to any excipients in the desvenlafaxine formulation. Concomitant use of PRISTIQ with products containing venlafaxine is not recommended.

Recent analyses of placebo-controlled clinical trial safety databases from selective serotonin reuptake inhibitors (SSRIS) and other newer antidepressants suggest that use of these drugs in patients under the age of 18 may be associated with behavioural and emotional changes, including an increased risk of suicide ideation and behaviour over that of placebo.

The small denominators in the clinical trial database, as well as the variability in placebo rates, preclude reliable conclusions on the relative safety profiles among the drugs in the class. There are clinical trial and post-marketing reports with SSRIs and other newer antidepressants, in both pediatrics and adults, of severe agitation-type events that include: akathisia, agitation, disinhibition, emotional lability, hostility, aggression and depersonalization. In some cases, the events occurred within several weeks of starting treatment.

Rigorous clinical monitoring for suicide ideation or other indicators of potential for suicide behaviour is advised in patients of all ages, especially when initiating therapy or during any change in dose or dosage regimen. This includes monitoring for agitation-type emotional and behavioural changes.

Patients currently taking PRISTIQ should NOT be discontinued abruptly, due to risk of discontinuation symptoms. At the time that a medical decision is made to discontinue an SSRI or other newer antidepressant drug, a gradual reduction in the dose, rather than an abrupt cessation is recommended.

Reference: 1. Pfizer Canada Inc. PRISTQQ Product Monograph, November 2010. Product Monograph available upon request.

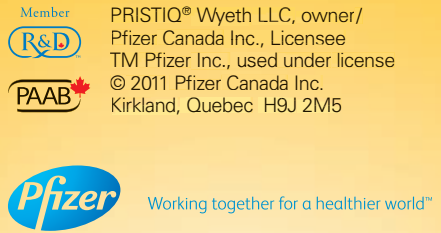

\section{Count on}

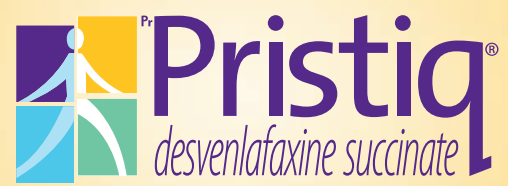

for powerful symptom relief 